\title{
Amorphous silicon as hydrogen glass
}

\author{
S C AGARWAL \\ Department of Physics, Indian Institute of Technology, Kanpur 208016, India
}

\begin{abstract}
The hydrogen in hydrogenated amorphous silicon (a-Si:H) makes it behave like a hydrogen glass. Above a temperature $T_{E}$, which is analogous to the glass transition temperature, the hydrogen is able to move more freely than below $T_{\mathbf{E}}$. This motion of hydrogen is believed to be responsible for the observed thermal and light induced metastabilities in a $\mathrm{Si}: \mathrm{H}$. However, the changes in the microstructure of the bonded hydrogen upon thermal quenching are found to influence the electronic properties of a-Si:H, in a manner, which is different from light soaking. Our studies suggest that the light soaking changes the potential Huctuations in lithium doped a-Si: $\mathrm{H}$, whereas the thermal quenching does not.
\end{abstract}

Keywords. Amorphous silicon; metastabilities; Staebler Wronski effect; thermal quenching; potential fuctuations; hydrogen glass model.

\section{Introduction}

It has been known for some time now that hydrogenated amorphous silicon $(\mathrm{a}-\mathrm{Si}: \mathrm{H})$, owes its sensitivity to dopants to the presence of hydrogen in it. In the most commonly used technique to prepare a-Si:H, silane gas $\left(\mathrm{SiH}_{4}\right)$ at low pressure is subjected to a high dc or RF electric field to produce a glow discharge. Under suitable conditions of substrate temperature, pressure, electric power etc. it is possible to make a-Si: $\mathrm{H} n$ type or $p$ type at will, by adding during preparation small amounts of either phosphine $\left(\mathrm{PH}_{3}\right)$ or diborane $\left(\mathrm{B}_{2} \mathrm{H}_{6}\right)$ to $\mathrm{SiH}_{4}$. It has enabled us to make relatively inexpensive solar cells, flat panel displays and other devices. It may be noted here that amorphous silicon prepared by other methods, e.g. vacuum evaporation or sputtering in argon atmosphere (so that it has no hydrogen or some other bond terminator) cannot be so doped. Amorphous silicon is in the form of a tetrahedral network containing heterogeneities in the form of voids which can be a few hundred angstrom in size as seen by transmission electron microscopy. The internal surfaces of these voids contain a large number of dangling bonds, whose density is estimated to be $\approx 10^{19} \mathrm{~cm}^{-3}$ in non-hydrogenated amorphous silicon. This high density of dangling bonds makes it impossible to dope a-Si without hydrogen. On the other hand, in a-Si: $\mathrm{H}$, the electrons in hydrogen pair up with the dangling bonds, thereby reducing the density of states in the gap to sufficiently low levels to allow doping. It is puzzling, however, that the amount of hydrogen present in the best quality (lowest defect density) a- $\mathrm{Si}$ : $\mathrm{H}$ is about 10 at. $\%$ which is much more than the number of dangling bonds. Although hand waving arguments, such as the possible relaxation of silicon network with larger amount of hydrogen have been given, the reason for such large concentrations of hydrogen required to produce good quality material is far from clear. (For reviews on a-Si:H, see for example Pankove 1984).

Apart from the difficulties in understanding the exact role played by hydrogen in improving the performance of amorphous silicon, it now seems that the presence of hydrogen may be responsible for the observed light and thermally induced metastabilities in a-Si:H. Upon exposure to visible light the amorphous silicon solar cells degrade and settle down to a value of efficiency, which is lower than the initial value. Electron 
spin resonance shows an increased density of dangling bonds in a-Si:H upon light soaking (Dersch et al 1981) and therefore it appears that hydrogen movement may be responsible for the same. Such a light-induced metastable change, commonly known as the Staebler Wronski effect, is reversible upon annealing at around $200^{\circ} \mathrm{C}$ for about an hour. (Staebler and Wronski 1977). Considerable work has been done on the study of this effect, because of its implications for the a-Si: $\mathrm{H}$ solar cells. It has been found that dark conductivity and photoconductivity decrease and subband gap absorption increases as new defects are created in bulk and on surface (Kumar and Agarwal 1984) and Fermi-level moves towards the middle of the mobility gap.

More recently, it has been discovered (Street et al 1986) that fast quenching from a temperature higher than the equilibration temperature $T_{E}$ results in an increased dark conductivity at room temperature in doped a- $\mathrm{Si}: \mathrm{H}$. This metastable state obtained by thermal quenching can also be annealed out at $T>T_{\mathrm{E}}$, in a fashion similar to the light soaked state, mentioned earlier. Further, $T_{\mathrm{E}}$ depends on the concentration and the kind of dopant chosen to dope a- $\mathrm{Si}: \mathrm{H}$.

Several questions arise, such as reasons for metastability in a-Si: $\mathrm{H}$, role of hydrogen, relationship of quenching to the Staebler Wronski effect, etc. In general, amorphous semiconductors are prepared by processes involving rapid quenching from the vapour or liquid phase and are, therefore, frozen in a state of metastable equilibrium. Annealing at high temperatures can induce transitions from one metastable state to another. Similarly, exposure to light (or other particles, e.g. electrons or ions) can also induce metastable changes. Thus $T_{\mathrm{E}}$ can be thought to be analogous to the glass transition temperature $T_{\mathrm{g}}$, observed in glasses, above which, the time taken to go from one structural configuration to another is shorter than the time scale of the experiment. In chalcogenide glasses the glass transition is usually visible as a step in a differential scanning calorimetry curve (DSC), when the specific heat increases for $T>T_{\mathrm{g}}$, as extra degrees of freedom become available to the system. In contrast, DSC curves for tetrahedrally bonded amorphous semiconductors (e.g., a-Ge or a-Si) do not show the glass transition so obviously. For example, Matsuo et al (1988) observe a peak (and not a step) in DSC curve in their fast quenched phosphorous doped a-Si: $\mathbf{H}$ thin films.

On the other hand, it has been suggested that a-Si: $\mathrm{H}$ is a hydrogen glass, where hydrogen moves more freely in a rigid Si network for $T>T_{\mathrm{E}}$ and $T_{\mathrm{E}}$ is analogous to $T_{\mathrm{g}}$. Measurements of diffusion constant of hydrogen in a-Si: $\mathrm{H}$ support this 'Hydrogen Glass Model' (Kakalios et al 1978).

We have tried to investigate whether it is the disorder or the hydrogen, which is responsible for these instabilities. We studied the quenching and light soaking effects in chalcogenide glasses and found that although some chalcogenides show light-induced metastabilities, none of the chalcogenides studied exhibited thermally induced effects. Thus it appears that the thermally induced metastabilities in $\mathrm{a}-\mathrm{Si}: \mathrm{H}$ are linked to the presence of hydrogen. In an attempt to see the effect of disorder we studied some a-Si: $\mathrm{H}$ after irradiation with $\mathrm{Kr}$ ions. The results show an increase in $T_{\mathrm{E}}$ and can be understood assuming that some hydrogen was lost irreversibly during irradiation.

There is still the question whether the light-induced and thermally-induced metastabilites are identical in nature. We measured the changes in dark conductivity $\sigma(T)$ and thermopower $S(T)$ after both light soaking and fast quenching of lithium doped $\mathrm{a}-\mathrm{Si}: \mathrm{H}$ films. The data was analysed according to the prescription given by Overhof and Beyer (1981). We have reached the conclusion that the two effects are not the same. In particular, we find that light soaking changes the potential fluctuations in the a-Si:H 
films significantly. In contrast, fast quenching leaves the potential fluctuations relatively unchanged.

\section{Metastabilities and the hydrogen glass model}

The results for light soaking (LS), and fast quenching (FQ) on the dark conductivity $(\sigma)$ of a lithium doped $\mathrm{a}-\mathrm{Si}: \mathrm{H}$ film are shown in figure 1 . The experimental details are given elsewhere(Agarwal et al 1993a, b). Here, it may suffice to note that the SC and FQ states are obtained by heating the sample to about $200^{\circ} \mathrm{C}$ and then cooling to room temperature at a slow rate $(1 \mathrm{~K} / \mathrm{min})$ or a fast rate $(400 \mathrm{~K} / \mathrm{min})$ respectively. Light soaking is done at room temperature for $15 \mathrm{~h}$ by exposing the sample to heat-filtered white light from a tungsten halogen lamp. The curves shown in figure 1 were obtained by measuring $\sigma$, while heating the sample in the SC, LS or FQ state. We see that light

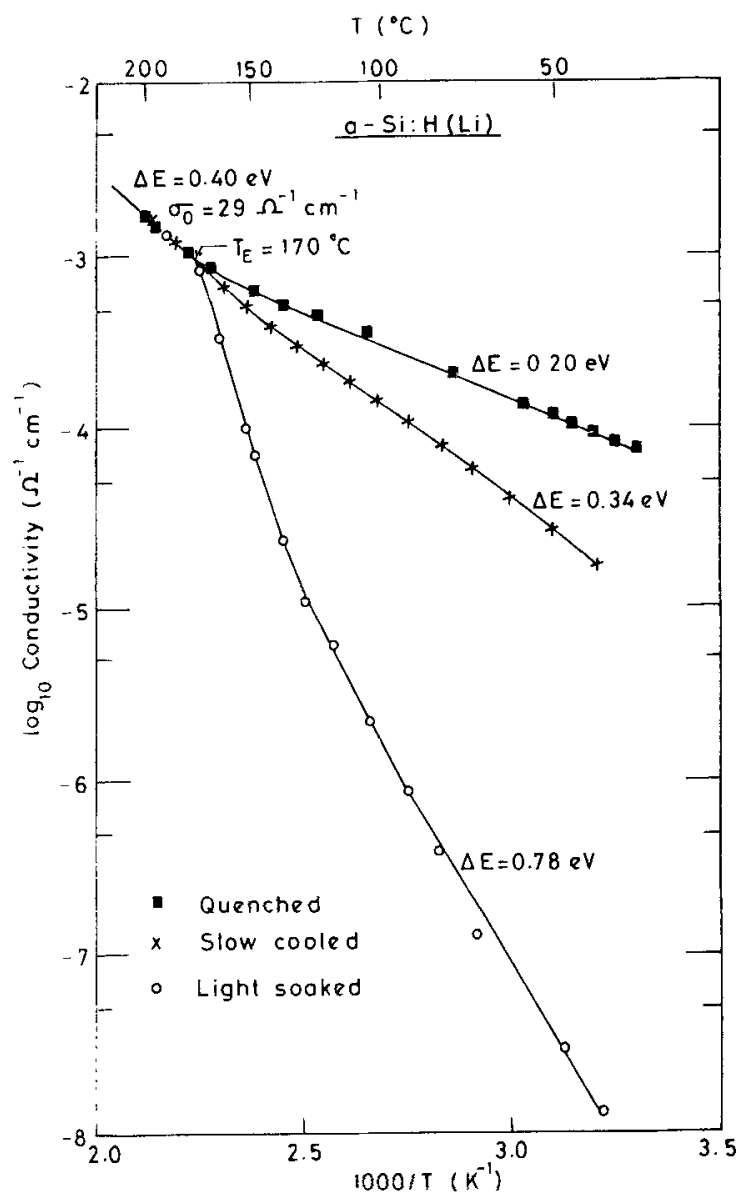

Figure 1. Effect of light soaking (circles) and fast quenching ( $400 \mathrm{~K} / \mathrm{min})$ (dark squares) on the dark conductivity of lithium doped a-Si:H. The slow cooled state has conductivity marked by crosses. Equilibration temperature $T_{\mathrm{E}}$ is the same $\left(170^{\circ} \mathrm{C}\right)$ for light soaked and quenched states (Agarwal et al 1993b). 
soaking (LS) decreases $\sigma$ at room temperature, as compared to the SC state, whereas fast quenching increases $\sigma$. Although the effect of LS and FQ are contrary, values of $\sigma(T)$ do merge at $T_{\mathrm{E}}=170^{\circ} \mathrm{C}$ and then follow a single curve for $T>T_{\mathrm{E}}$.

The LS and FQ states relax slowly to the SC state as a function of time at fixed $T$. Such relaxation curves are shown in figures 2 and 3. In both LS and FQ states, they follow a stretched exponential of the form

$$
y(t)=\exp \left[-(t / \tau)^{\beta}\right]
$$

where

$$
y(t)=[\sigma(t)-\sigma(\infty)] /[\sigma(0)-\sigma(\infty)]
$$

These results are quite similar to those reported on $\mathrm{P}$ and $\mathrm{B}$ doped samples (Kakalios et al 1987). The results after fast quenching have been explained in the literature on the basis of the so called hydrogen glass model (Kakalios et al 1987). According to it, hydrogen diffuses in a rigid matrix of silicon more freely for $T>T_{\mathrm{E}}$ than for $T<T_{\mathrm{E}}$. Quenching freezes the a-Si: $\mathrm{H}$ sample in a metastable state of equilibrium. Since $y(t)$ is a measure of the number of frozen-in band-tail carriers, it is expected to follow the rate equation:

$$
(\mathrm{d} y(t) / \mathrm{d} t)=C y(t)
$$

If $C$ in (2) has a power law time dependence

$$
C \propto t^{-x}
$$

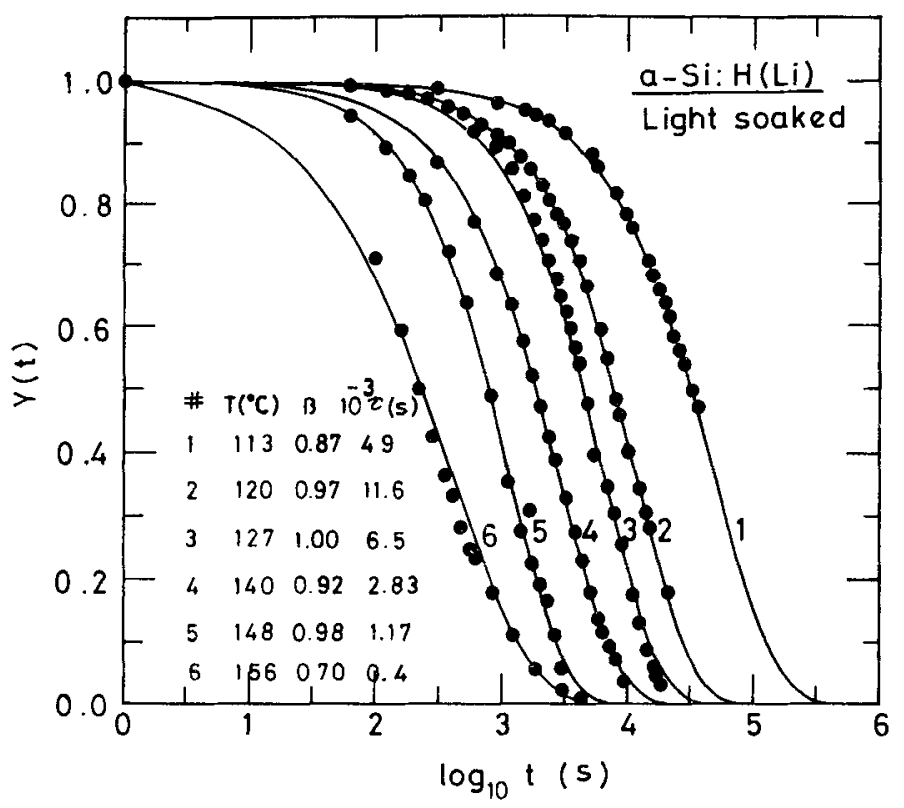

Figure 2. Relaxation of the lithium doped a-Si:H. from the light soaked state at various temperatures as indicated. $\beta$ and $\tau$ are parameters that fit the data to a stretched exponential (Agarwal 1995). 


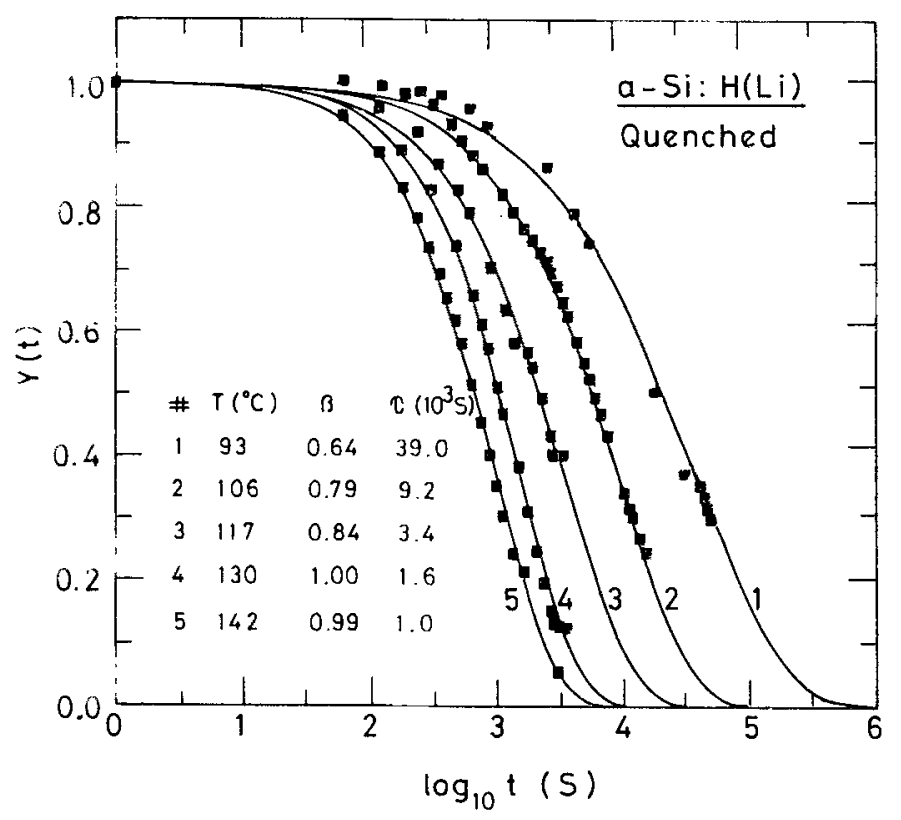

Figure 3. Stretched expunential fit to the relaxation of the a-Si:H (Li) sample from the fast quenched state at various fixed temperatures (Agarwal 1995).

the integration of (2) yields a stretched exponential. A comparison with (1) yields $\beta=1-\alpha$. The diffusion constant of hydrogen $\left(D_{\mathrm{H}}\right)$ which is expected to be proportional to $C$ has been measured at different temperatures using deuterium tracer as monitored by SIMS (Street et al 1987). It is found that $D_{\mathrm{H}} \alpha t^{-\alpha}$ and that $\alpha+\beta \approx 1$. Since $\alpha$ and $\beta$ are measured in two independent experiments, it lends credence to the hydrogen glass model.

In order to check further the validity of the hydrogen glass model, we performed the quenching experiments on phosphorous doped $\mathrm{a}-\mathrm{Si}: \mathrm{H}$ before and after irradiation with $1 \cdot 1 \mathrm{MeV} \mathrm{Kr}^{++}$ions $\left(10^{16} / \mathrm{cm}^{2}\right)$ (Agarwal et al 1991). The irradiated sample seems to loose hydrogen and as expected (Battezzati et al 1991) $T_{\mathrm{E}}$ increases as shown in figure 4.

As already remarked, the glass transition in a-Si: $\mathrm{H}$ is not clearly seen by DSC experiments (Matsuo et al 1988). But in similar experiments on chalcogenides one can clearly identify this transition. We chose two chalcogenides $\mathrm{Ge}_{15} \mathrm{Se}_{85}\left(T_{\mathrm{g}} \approx 125^{\circ} \mathrm{C}\right)$ and $\mathrm{Ge}_{22} \mathrm{Se}_{68} \mathrm{Ag}_{10}\left(T_{\mathrm{g}} \approx 135^{\circ} \mathrm{C}\right)$ with activation energies $\approx 0.4-0.5 \mathrm{eV}$. These parameters are similar to those of doped a-Si:H. Thin films had a different activation energy, probably because of different composition. Both bulk as well as thin films were studied. No quenching effect was, however, seen (Agarwal et al 1993a), although quenching rates similar to a-Si: $\mathrm{H}$ were used (see figure 5). This can be understood if one argues that the presence of hydrogen is necessary for the observation of quenching effects.

\section{Do light soaking and quenching affect $\mathbf{a}-\mathrm{Si}: \mathrm{H}$ in the same manner?}

We finally address ourselves to the question, whether the changes caused by light soaking are the same as those created by quenching. It has been suggested that the 


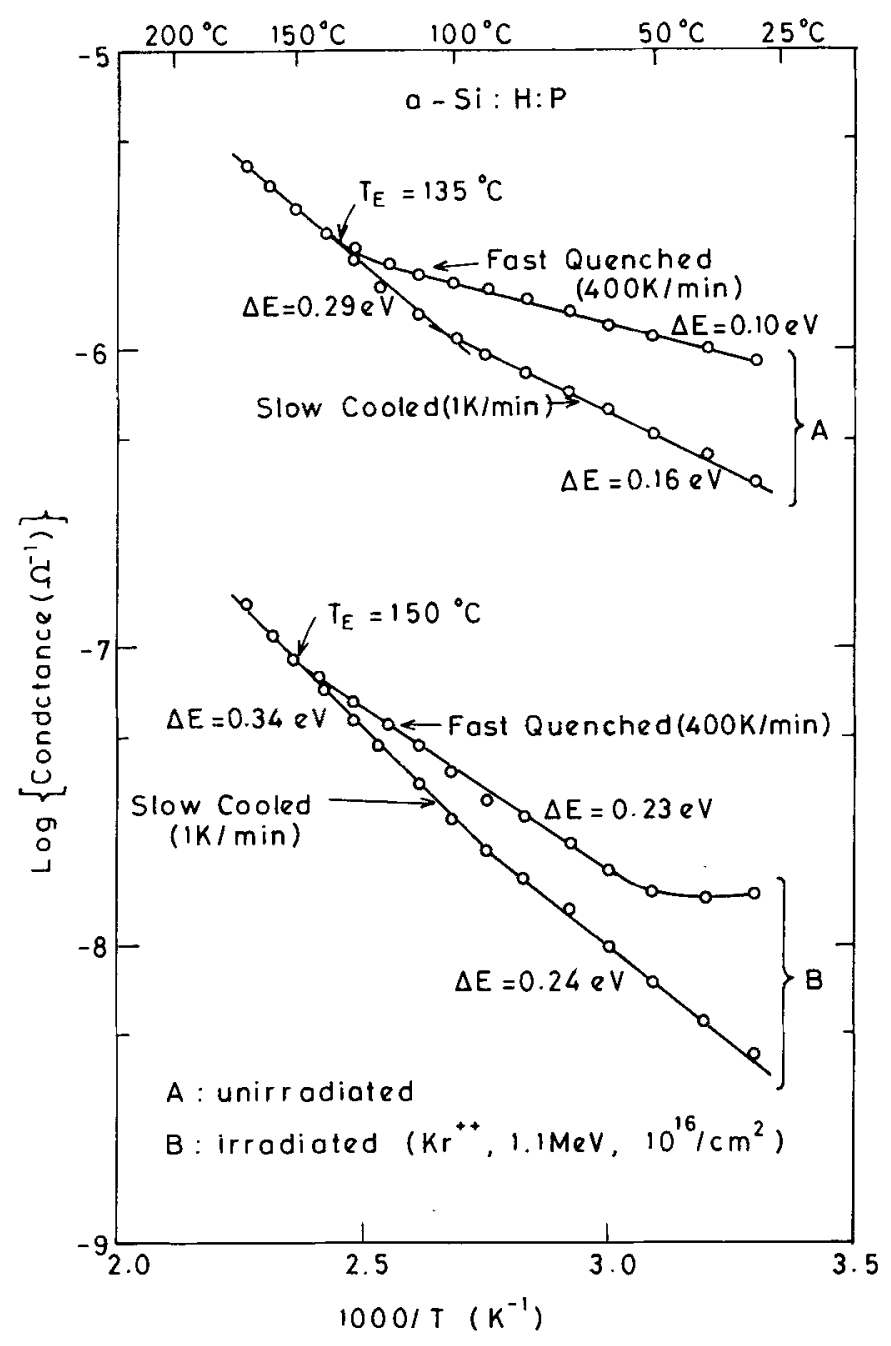

Figure 4. Effect of fast quenching on the dark conductance for a phosphorous doped a-Si:H sample, before $(A)$ and after $(B)$ irradiation with $1 \cdot 1 \mathrm{MeV} \mathrm{Kr}{ }^{++}$ions $\left(10^{16} / \mathrm{cm}^{2}\right)$. $T_{\mathrm{E}}$ increases for the irradiated sample (Agarwal et al 1991).

motion of hydrogen is responsible for both types of instabilites. Indeed, if the direction of change in $\sigma$, which is opposite in the two cases; is ignored, the same value of $T_{\mathrm{E}}$ and the stretched exponential decays, as described above, tempt one to conclude that the two effects are related and probably affect the sample in the same manner.

A closer look, however, reveals a different story. Figure 6 shows that the parameter $\tau$ is thermally activated with different activation energies for the light soaked (LS) and thermally quenched $(\mathrm{FQ})$ states. If they had both been relaxing through the same process, the activation energies should have been the same. The temperature dependence of the parameter $\beta$ for the two types of instabilities is shown in figure 7 .

Finally, figures 8 and 9 show our measurement of $\sigma$ and thermopower on another a-Si: $\mathrm{H}$ (Li) sample (doped with a higher concentration of $\mathrm{Li}$, than that of figure 1 ) in 


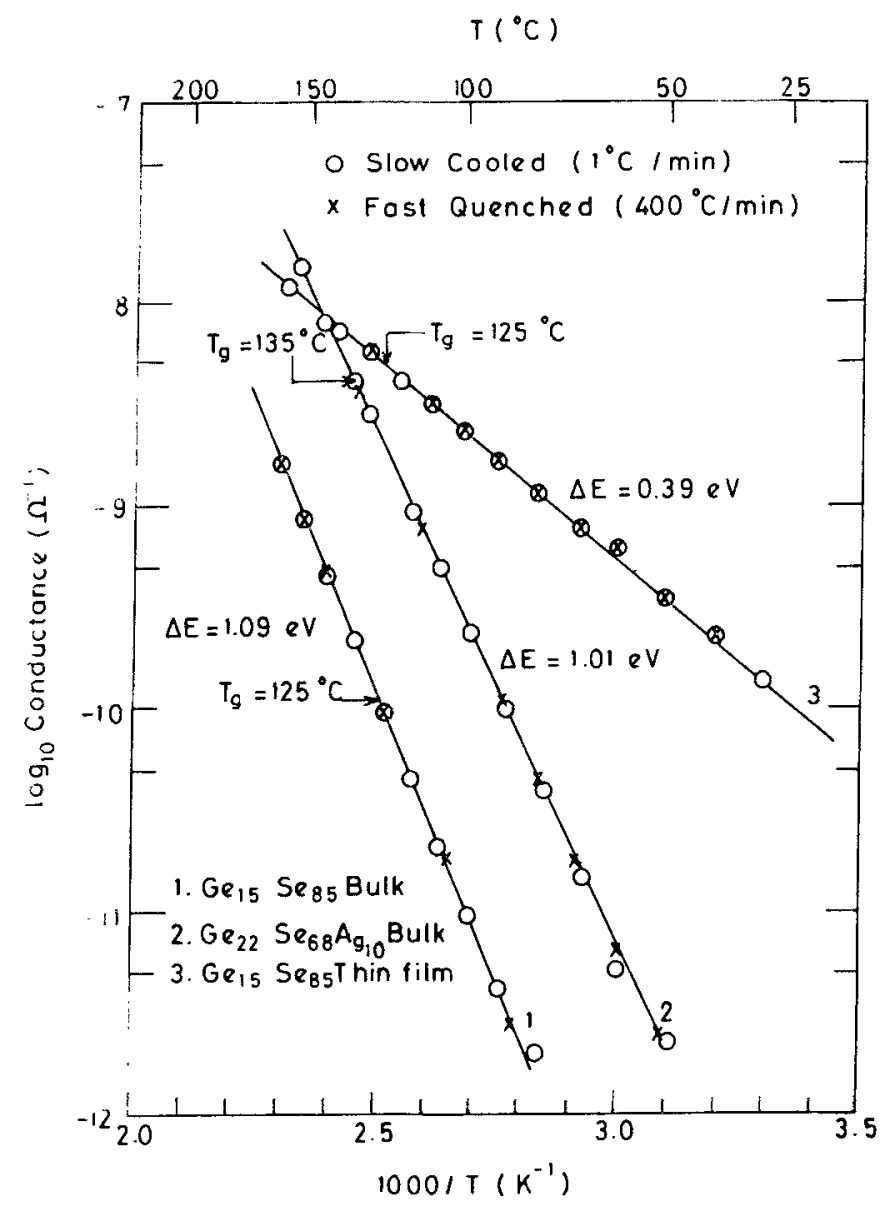

Figure 5. Lug $\mathrm{G}$ vs $1000 / T$ for chalcogenude glasses in thin film and bulk form, showing no effect of quenching. $T_{\mathrm{g}}$ is the glass transition temperature, measured by DSC on bulk specimen (Agarwal et al 1993a).

the SC, LS, and FQ states. For band conduction it can be shown that thermopower $S$ follows the following temperature dependence (Fritzsche 1971):

$$
S(T)=-(e / k)\left[\left(E_{\mathrm{c}}-E_{\mathrm{f}}\right) / k T+A(T)\right]
$$

where $A(T)$ is a constant of the order of unity and $E_{\mathrm{c}}$ and $E_{\mathrm{f}}$ are the energies of the conduction band edge and the Fermi level respectively. Since $\left(E_{\mathrm{c}}-E_{\mathrm{f}}\right)$ can be temperature dependent because $E_{\mathrm{f}}$ may show a statistical shift. Overhof and Beyer (1981) have suggested that it is better to work with $Q$, which is a combination of $S$ and $\sigma$ :

$$
\begin{aligned}
& \sigma=\sigma_{0} \exp \left[-\left(E_{\mathrm{c}}-E_{\mathrm{f}}\right) / k T\right], \\
& Q=\ln \sigma+|e S / k|=\ln \sigma_{0}+A(T)=Q_{0}+E_{Q} / k T .
\end{aligned}
$$

Overhof and Beyer (1981) have shown that $E_{Q}$, the slope of $Q$ vs $1 / T$, is related to the potential fluctuations in the material. These potential fluctuations in a-Si:H may be 


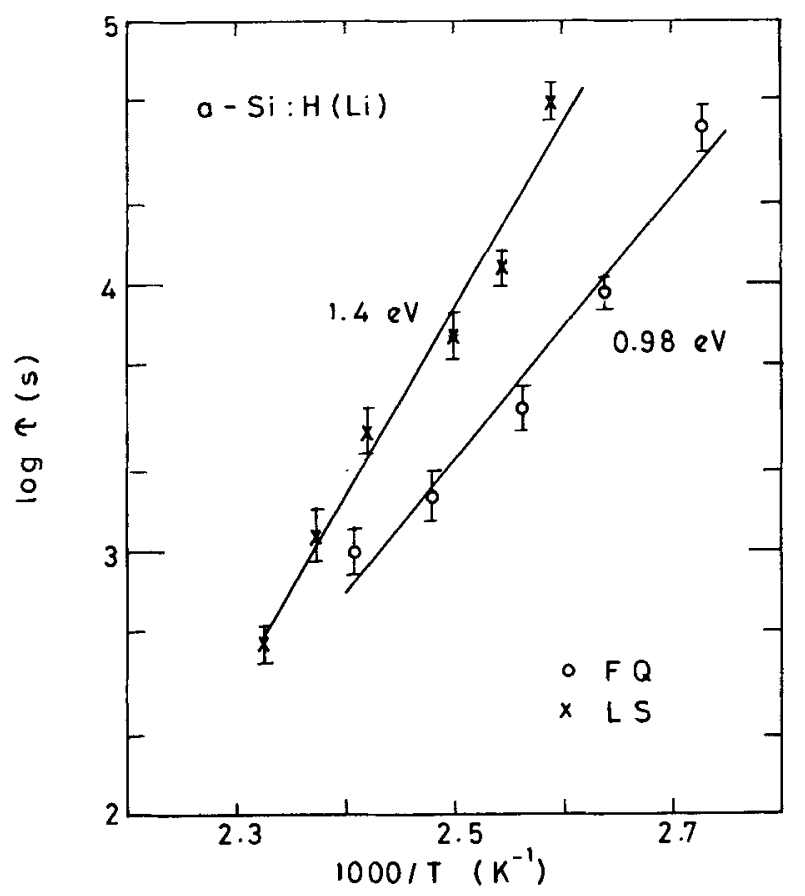

Figure 6. $\log \tau$ vs $1 / T$ plot for the relaxation of an a-Si:H (Li) sample in light soaked LS and fast quenched FQ states. Note the different activation energies in the two cases (Agarwal 1995).

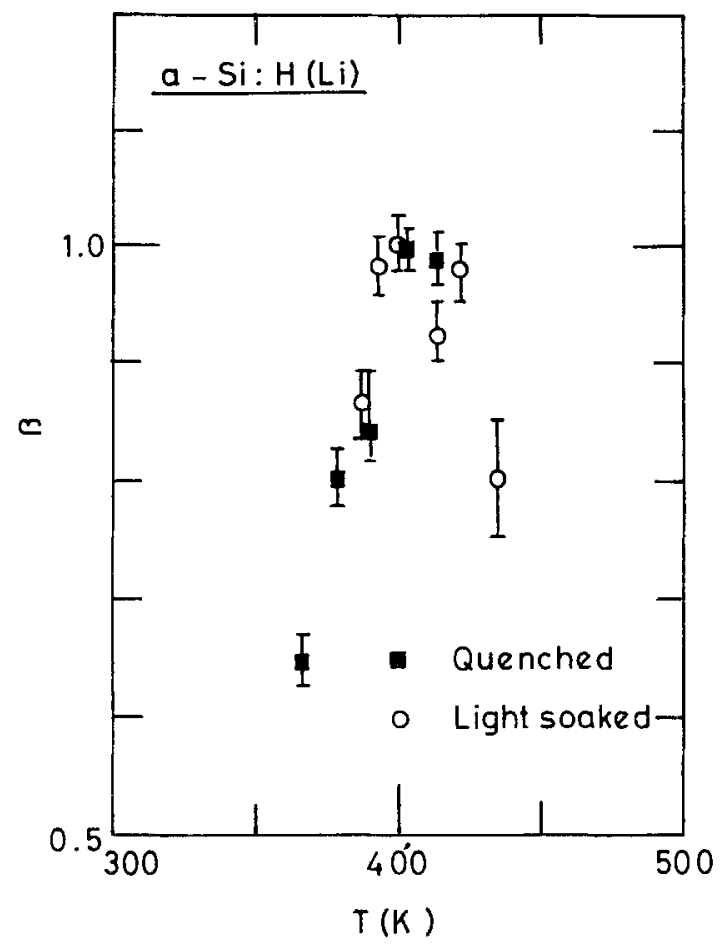

Figure 7. Variation of the parameter $\beta$ with $T$ obtained by fitting the relaxation curves of the a-Si:H (Li) sample from the light soaked (O) and quenched ( $\square$ ) states (Agarwal 1995). 


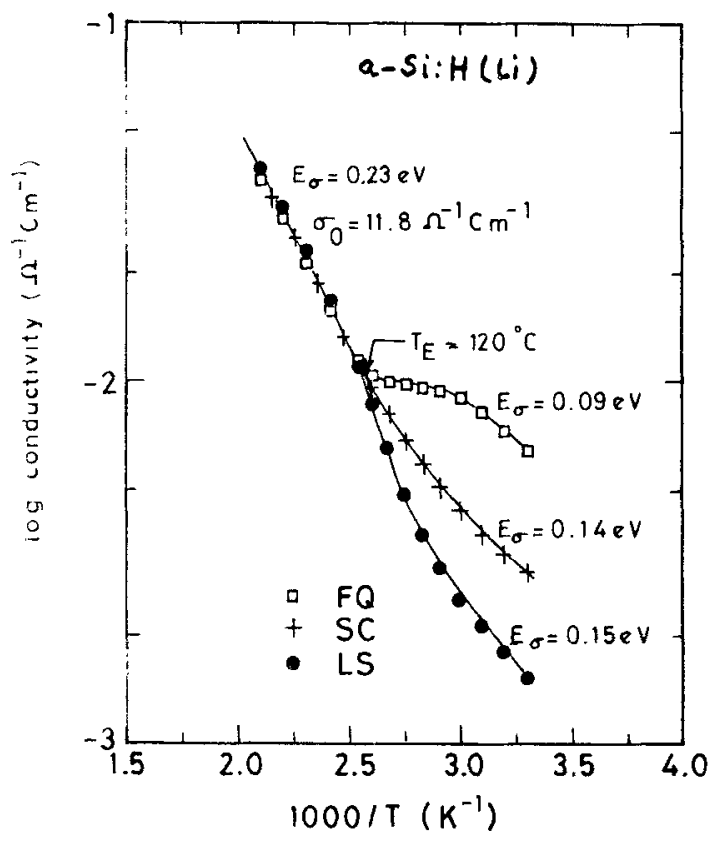

Figure 8 . Eflect of light soaking and fast quenching on the dark conductivity of a- $\mathrm{Si}: \mathrm{H}(\mathrm{Ll})$, with a higher doping concentration of lithium. Notice that $T_{\mathrm{E}}$ is the same for the two cases A Agarwal 1995).

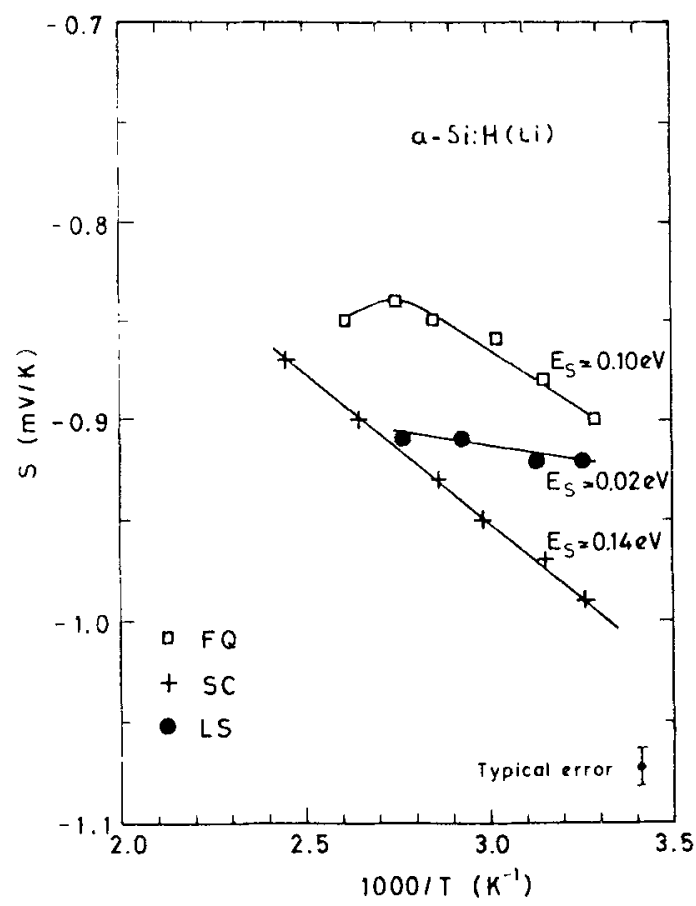

Figure 9. Thermopower (S) of the a- $\mathrm{Si}: \mathrm{H}(\mathrm{Li})$ sample of figure 8, in the slow cooled (SC). light soaked (LS) and fast quenched states (FQ). Typical error bar in the measurement is also shown Agarwal 1995). 


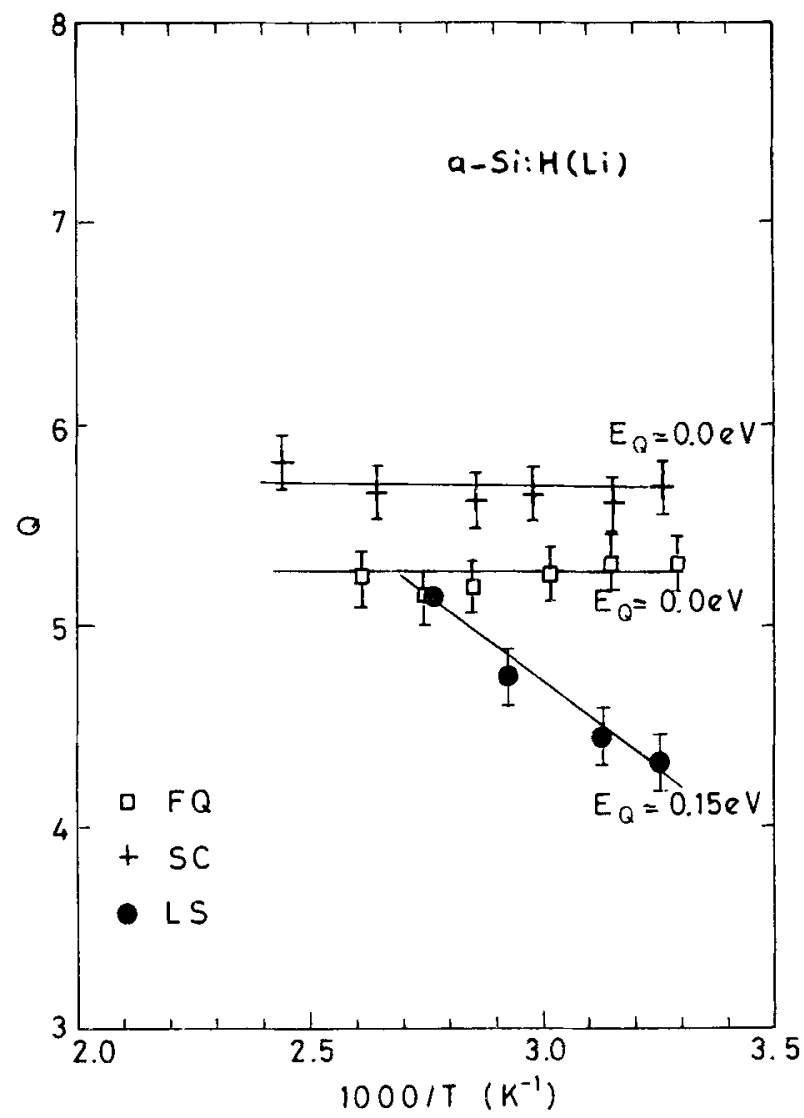

Figure 10. Function $Q$ of (3) in the slow cooled (SC). light soaked (LS) and fast quenched states (FQ). The slope $E_{Q}$ is almost zero for the SC and FQ states, whereas for LS state it is about $0.15 \mathrm{eV}$ (Agarwal 1995).

caused by heterogeneities present in the material. In addition, the donor impurities may also make some contribution (Howard and Street 1991).

Figure 10 shows the plot of $Q$ vs $1 / T$ in the SC. LS and FQ states. The slope $E_{Q}$ is negligible for the $\mathrm{SC}$ and $\mathrm{FQ}$ states but has a value $\approx 0.15 \mathrm{eV}$ in the LS state. Thus, light soaking and quenching affect the lithium doped a-Si: $\mathrm{H}$ differently. Light soaking changes the potential fluctuations whereas the quenching does not.

\section{Summary and conclusions}

We have seen that hydrogen plays an important role in making the amorphous silicon amenable to doping, thus making it useful for device fabrication. On the other hand, it appears to play a significant role in the observed light and thermally induced metastabilities in a-Si: $\mathrm{H}$. The equilibration temperatures $T_{\mathrm{E}}$ for the light soaked and thermally quenched lithium doped a-Si: $\mathrm{H}$ are found to be equal. Further, relaxation as a function of time at fixed temperature is found to obey a stretched exponential in both cases. Also, this is very similar to the behaviour of thermally quenched $\mathrm{P}$ doped a-Si:H. 
It. therefore, seems that the "hydrogen glass model' may explan both the effects. In this model, hydrogen diffuses in a rigid network of silicon. becoming considerably more mobile for $T>T_{\mathrm{f}}$ than for temperatures below $T_{\mathrm{F}}$. The absence of thermal quenching effects in chalcogenide glasses containing no hydrogen might be taken as an evidence in lavour of the hydrogen glass model.

Although there are many similarities in the light induced and thermally induced metastabilities there are several differences too which need to be pointed out. Apart from the different direction of change in $\sigma$ of the SC state after LS and FQ, the relaxation parameters $\tau(T)$ and $\beta(T)$ show different behaviours as functions of temperature in the two cases. Using thermopower and the conductivity data and the analysis of Overhof and Beyer (1981) it is found that potential fluctuations change on light-soaking whereas the quenching leaves them unchanged (Agarwal 1995).

It may be argued that the absence of quenching effects in chalcogenide glasses (Agarwal et al 1993a) might be linked with these being the lone pair semiconductors. This is in contrast to a-Si:H which is tetrahedrally bonded. However, metastable changes in conductivity (Agarwal and Fritzsche 1974) and shift in the optical absorption edge (Pfeiffer et al 1991) in chalcogenide glasses created by light exposure, are well known. On the other hand. no reports of quenching effects in chalcogenide glasses have a ppeared in the literature so far. Can we then conclude that hydrogen is really necessary for observing quenching effects? Probably not. In hydrogen glass model for a- $\mathrm{Si}: \mathrm{H}$, a rigid network of silicon is necessary in which hydrogen is more mobile above $T_{\mathrm{E}}$. No such rigid network exists in chalcogenide glasses above $T_{g}$. This might explain the absence of quenching effects in chalcogenide glasses.

Before closing this discussion. I would like to point out that there are now reports of a- $\mathrm{Si} ; \mathrm{H}$ films prepared by $\mathrm{SiH}_{4}+\mathrm{He}$, which are quite stable against quenching (Meaudre et al 1991) although they contain more hydrogen than the regular a-Si:H films prepared from pure $\mathrm{SiH}_{4}$, which show the same effect. Obviously, a lot more remains to be done to understand better some of these puzzles.

\section{Acknowledgements}

I would like to thank Ms Pratima Agarwal who carried out most of the experimental work. I am also grateful to my students and colleagues, Mr Nazrul Islam, Dr Santosh Kumar. Dr Satyendra Kumar, Ms Manju Malhotra, Mr Anil Sinha. Mr Alok Srivastava and Dr S K Tripathi for many valuable discussions and help. The financial assistance provided by the Department of Science and Technology, New Delhi is also gratefully acknowledged.

\section{References}

tgarwal P 1995 thermal and lighn induced motassablhties in phosphorous and lithium doped hydrogenated amorphous silicom. Ph.D. Thesis, Physics Department, IIT Kanpur

Agarwal P. Agarwal S C. Bhattacharya E and Dixit P N 1991 SSP Symposium (DAE) C34 222

Agarwal P. Bhattacharya E, Kumar S and Kumar A 1993a Indian J. Pure \& Appl. Phys. 31367

Agarwal P. Tripathi S K, Kumar S and Agarwal S C 1993b SSP Symposium (DAE) C36 84

Agarwal S C and Fritzsche H 1974 Phys. Rev, B10 435

Battezzati L., Demichelis F, Pirri C F. Tagliaferro A and Tresso E $1991 \mathrm{~J}$. Non-Cryst. Solids 137113887

Dersch H. Stuke J and Beichler J 1981 Appl. Phys. Lett. 38456 
Fritzsche H 1971 Solid State Commun. 91813

Howard J A and Street R A 1991 Phys. Rev. B44 7935

Kakalios J, Street R A and Jackson W B 1987 Phys. Rev. Lett. 591037

Kumar S and Agarwal S C 1984 Philos. Mag. B49 L53

Matsuo S, Nasu H, Akamatsu C, Hayashi R, Imura T and Osaka Y 1988 Jap. J. Appl. Phys. 27 L132

Meaudre R, Meaudre M, Rocai Cabarrocas P, Tanidi S, Bouizems Y and Theye M L 1991 J. Non-Cryst. Solids $137 / 138171$

Overhof $\mathrm{H}$ and Beyer W 1981 Philos. Mag. B43 433

Pankove J 1984 (ed), Semiconductors and semimetals (New York: Academic Press) Vol. 21

Pfeiffer G, Paesler M A and Agarwal S C 1991 J. Non-Cryst. Solids 130111

Staebler D L and Wronski C R 1977 Appl. Phys. Lett. 31292

Street R A, Kakalios J and Hayes T M 1986 Phys. Rev. B34 3030

Street R A, Tsai C C, Kakalios J and Jackson W B 1987 Philos. Mag. B56 305 